\title{
Plasma osteoprotegerin levels predict cardiovascular and all-cause mortality and deterioration of kidney function in type 1 diabetic patients with nephropathy
}

\author{
A. Jorsal • L. Tarnow • A. Flyvbjerg • H.-H. Parving • \\ P. Rossing • L. M. Rasmussen
}

Received: 10 January 2008 / Accepted: 16 July 2008 / Published online: 22 August 2008

(C) Springer-Verlag 2008

\begin{abstract}
Aims/hypothesis The bone-related peptide osteoprotegerin is produced by vascular cells and is involved in the process of vascular calcification. The aim of this study was to investigate the predictive value of plasma levels of osteoprotegerin in relation to mortality, cardiovascular events and deterioration in kidney function in patients with type 1 diabetes.

Methods This prospective observational follow-up study included 397 type 1 diabetic patients with overt diabetic nephropathy (243 men; age [mean $\pm \mathrm{SD}] 42.1 \pm 10.6$ years,
\end{abstract}

A. Jorsal $(\bowtie) \cdot$ L. Tarnow $\cdot$ P. Rossing

Steno Diabetes Center,

Niels Steensens Vej 2,

2820 Gentofte, Denmark

e-mail: ajrs@steno.dk

\section{A. Flyvbjerg}

The Medical Research Laboratories, Clinical Institute and Medical Department M (Diabetes and Endocrinology),

University of Aarhus,

Aarhus, Denmark

H.-H. Parving

Department of Medical Endocrinology, Rigshospitalet,

University Hospital of Copenhagen,

Copenhagen, Denmark

H.-H. Parving

Faculty of Health Science, University of Aarhus,

Aarhus, Denmark

L. M. Rasmussen

Department of Clinical Biochemistry, Odense University Hospital, University of Southern Denmark,

Odense, Denmark duration of diabetes $28.3 \pm 9.9$ years, GFR $67 \pm 28 \mathrm{ml} \mathrm{min}^{-1}$ $1.73 \mathrm{~m}^{2}$ ) and a group of 176 patients with longstanding type 1 diabetes and persistent normoalbuminuria (105 men; age $42.6 \pm 9.7$ years, duration of diabetes $27.6 \pm 8.3$ years). Results The median (range) follow-up period was 11.3 $(0.0-12.9)$ years. Among patients with diabetic nephropathy, individuals with high osteoprotegerin levels (fourth quartile) had significantly higher all-cause mortality than patients with low levels (first quartile) (covariate-adjusted hazard ratio [HR] 3.00 [1.24-7.27]). High osteoprotegerin levels also predicted cardiovascular mortality (covariateadjusted HR 4.88 [1.57-15.14]). Furthermore, patients with high osteoprotegerin levels had significantly higher risk of progression to end-stage renal disease than patients with low levels (covariate-adjusted HR 4.32 [1.45-12.87]). In addition, patients with high levels of plasma osteoprotegerin had an elevated rate of decline in GFR.

Conclusions/interpretation High levels of osteoprotegerin predict all-cause and cardiovascular mortality in patients with diabetic nephropathy. Furthermore, high levels of osteoprotegerin predict deterioration of kidney function towards end-stage renal disease.

Keywords Cardiovascular events · Diabetes · Diabetic nephropathy $\cdot$ End-stage renal disease $\cdot$ Glomerular filtration rate $\cdot$ Mortality $\cdot$ Osteoprotegerin

$\begin{array}{ll}\text { Abbreviations } \\ \text { ESRD } & \text { end-stage renal disease } \\ \text { HR } & \text { hazard ratio } \\ \text { UAER } & \text { urinary albumin excretion rate } \\ \text { NT-proBNP } & \begin{array}{l}\text { N-terminal prohormone brain } \\ \text { natriuretic peptide }\end{array}\end{array}$




\section{Introduction}

Despite improvements achieved during recent years in the treatment of type 1 diabetic patients, the long-term prognosis for these patients is still considerably worse than among individuals without diabetes. This unfortunate fact is founded on a high incidence of cardiovascular disease and end-stage renal disease (ESRD), due to the development of diabetic angiopathy.

Osteoprotegerin is present in bone and has moreover been found to play an important role in the vasculature. The molecule was discovered in 1997, where it was shown to function as a strong inhibitor of osteoclast formation in bone $[1,2]$. The peptide is a soluble TNF-receptor-like molecule without a membrane-spanning part [3]. It has been proposed that osteoprotegerin may be involved in the development of calcifications in the arterial wall, where the molecule is present in concentrations comparable with those in bone [4]. Supporting this idea, studies have shown that expression of the gene encoding osteoprotegerin and production of the protein are altered in arterial areas with calcification [5]. However, the exact functions of osteoprotegerin in relation to vascular calcifications and other arterial functions are not yet well defined [6-9]. Interestingly, we have previously observed that osteoprotegerin accumulates in human aortic tissue from patients with type 1 and type 2 diabetes, a finding that has very recently been confirmed in studies of experimental models of diabetic arteriopathy $[10,11]$.

Osteoprotegerin can be detected in plasma, but at present there is no information about the predominant source of circulating osteoprotegerin and nothing is known about its elimination from the blood. Plasma levels are increased in patients with diabetes $[12,13]$ as well as in non-diabetic individuals with coronary artery disease $[12,14,15]$. Of particular interest, the plasma osteoprotegerin concentration correlates to the degree of arterial calcification [16, 17], a phenomenon often observed in patients with diabetes [16, 17]. Furthermore, a relationship between increased plasma osteoprotegerin concentration and micro- and macrovascular parameters has been observed in patients with type 1 [18] and type 2 diabetes [13]. In addition, a few recent studies indicate that plasma osteoprotegerin may be a strong and independent predictor of cardiovascular events in some populations. This has been shown to be the case in patients with type 2 diabetes [19], as well as in non-diabetic patients after kidney transplantation [20] and after myocardial infarction [21].

In this paper, we extend previously observed correlations between plasma osteoprotegerin and cardiovascular risk factors in type 1 diabetic individuals with data on the capacity of plasma osteoprotegerin to predict death, cardiovascular events and ESRD through more than 11 years of follow-up.

\section{Methods}

Patients During 1993, 198 type 1 diabetic patients with diabetic nephropathy, whose GFR had been measured during the same year, were recruited from the outpatient clinic at Steno Diabetes Center for a case-control study. Simultaneously, a group of 192 patients with long-lasting type 1 diabetes and persistent normoalbuminuria were recruited as controls. Since then, all type 1 diabetic patients newly referred for measurement of kidney function have been invited to participate in an ongoing study of the genetics of diabetic nephropathy, and thus the original cohort of type 1 diabetic patients with diabetic nephropathy was expanded to 408 patients.

Plasma osteoprotegerin levels were determined and follow-up data were available for 397 (97\%) patients with diabetic nephropathy and 176 (92\%) controls.

Type 1 diabetes was considered present if the age at onset of diabetes was $\leq 35$ years and time to definite insulin therapy was $\leq 1$ year. Established diabetic nephropathy was defined by persistent albuminuria $(>300 \mathrm{mg} / 24 \mathrm{~h})$ in two out of three consecutive measurements in sterile urine samples, the presence of retinopathy and the absence of other kidney or urinary tract diseases [22]. In patients without retinopathy $(n=6)$ the diagnosis of diabetic glomerulopathy was verified by a kidney biopsy. Controls were patients with persistent normoalbuminuria $(<30 \mathrm{mg} /$ $24 \mathrm{~h}$ ) after at least 15 years of type 1 diabetes in patients not treated with ACE inhibitors or angiotensin II receptor blockers.

Baseline clinical and laboratory investigations All patients had blood samples and phenotypic characteristics collected as part of the EURAGEDIC project [23]. BP was measured twice in the resting state. Plasma lipid levels were determined from venous samples by standard methods. $\mathrm{HbA}_{1 \mathrm{c}}$ was determined by standard HPLC techniques with normal values in the range 4.1-6.4\%. Urinary AER (UAER) was measured in $24 \mathrm{~h}$ urine collections by an enzyme immunoassay [24]. Serum creatinine concentration was determined by a modification of Jaffe's method. GFR was measured annually in patients with diabetic nephropathy after a single injection of $3.7 \mathrm{MBq}{ }^{51} \mathrm{Cr}$-labelled EDTA by determination of radioactivity in venous blood samples taken 180, 200, 220 and 240 min after injection [25]. Linear regression analysis of serial GFR determinations in each individual was used to estimate the rate of decline in kidney function with time ( $\Delta$ GFR). ESRD was defined as kidney transplantation or dialysis. Diabetic retinopathy was assessed by fundus photography after pupillary dilatation and graded nil, simplex and proliferative retinopathy, respectively. Based on standardised questionnaires, current smokers of one or more cigarettes, cigars 
or pipes per day were classified as smokers and all others as non-smokers. Cardiovascular disease was considered present at baseline in patients with a history of admission for stroke or myocardial infarction. Plasma osteoprotegerin was measured by a sandwich ELISA using commercially available antibodies (R\&D Systems, Minneapolis, MN, USA), as previously described [13]. Briefly, mouse antihuman osteoprotegerin (R\&D Systems) was used as capture antibody and a biotinylated goat anti-human osteoprotegerin (R\&D Systems) for detection. Recombinant human osteoprotegerin was used for calibration and the analytical range of the assay was $62.5-4,000 \mathrm{ng} / \mathrm{ml}$. Plasma samples were diluted $1 / 3$ and measured in duplicate. The intra-assay $\mathrm{CV}$, as judged from duplicate measurements, was $3 \%$. The interassay variation was $8 \%(n=18$ control samples) in the present series of analysis. The samples were analysed in random order. Repeated freeze-thawing did not have any effect on results.

Follow-up In a prospective observational study design, the patients were followed until an endpoint was reached: the last visit to the Steno Diabetes Center or 1 September 2006. The primary endpoints were all-cause mortality, cardiovascular mortality and major cardiovascular events; secondary endpoints were progression of nephropathy ( $\Delta$ GFR and ESRD).

All patients were traced through the national register during autumn 2006. If a patient died before 1 September 2006 , the date of death was recorded and information on cause of death was obtained from the death certificate. Two observers reviewed all death certificates independently and the primary cause of death was recorded. Additional available information from necropsy reports was included. All deaths were classified as cardiovascular deaths unless an unequivocal non-cardiovascular cause was established [26]. Information about date of ESRD and non-fatal cardiovascular events were obtained from patient records or discharge letters from other hospitals. Major cardiovascular events were diagnosed as stroke, myocardial infarction, coronary artery bypass graft and/or percutaneous coronary intervention.

The study was performed in accordance with the Declaration of Helsinki, the local ethics committee approved the study and all patients gave their informed consent.

Statistical analysis Normally distributed variables are given as mean $\pm \mathrm{SD}$, whereas non-normally distributed variables were log-transformed before analysis and are given as median (range). Comparisons between groups were performed by unpaired Student's $t$ test or ANOVA. The $\chi^{2}$ test was used to compare non-continuous variables. A twotailed $p$ value of $\leq 0.05$ or less was considered statistically significant.
Log osteoprotegerin concentration was normally distributed and patients were divided into quartiles on the basis of the distribution of osteoprotegerin values. All time-toendpoint variables were analysed using a log-rank test and displayed on Kaplan-Meier plots as 1 minus cumulative survival. Cox regression models were used to estimate the unadjusted and adjusted hazard ratios with $95 \%$ CI. When analysing primary endpoints, we adjusted for the following covariates at baseline: sex, age, smoking, systolic BP, $\mathrm{HbA}_{1 \mathrm{c}}$, GFR, serum cholesterol, UAER, antihypertensive treatment and history of cardiovascular events. In an additional analysis we also adjusted for C-reactive protein and N-terminal prohormone brain natriuretic peptide (NTproBNP). When analysing data on ESRD and $\triangle$ GFR, we performed adjustment for sex, age and well-known progression promoters including systolic $\mathrm{BP}, \mathrm{HbA}_{1 \mathrm{c}}$, serumcholesterol, GFR, UAER and antihypertensive treatment. All covariates were entered in the Cox regression model when adjustments were performed. Areas under the receiver operating characteristic curves and their 95\% CIs were estimated.

All calculations were performed using a commercially available program (SPSS for Windows, version 14.0; SPSS, Chicago, IL, USA).

\section{Results}

Baseline characteristics The study was a prospective observational follow-up study with a median follow-up time until endpoint or last visit of $11.3(0.0-12.9)$ years. The study population included two groups: 397 patients with type 1 diabetes and diabetic nephropathy and 176 controls with type 1 diabetes for more than 15 years and persistent normoalbuminuria. Baseline clinical and laboratory characteristics of the 573 patients are shown in Table 1 . Patients with diabetic nephropathy received more insulin and antihypertensive medication, had higher BMI, $\mathrm{HbA}_{1 \mathrm{c}}$, $\mathrm{BP}$, serum creatinine, total cholesterol, serum triacylglycerol and osteoprotegerin levels but lower concentrations of HDL than patients with type 1 diabetes and normoalbuminuria $(p<0.05)$. On average, GFR was well preserved in patients with diabetic nephropathy.

Follow-up data Osteoprotegerin was measured in 176 patients with normoalbuminuria (2.6 (1.4-8.2) $\mu \mathrm{g} / \mathrm{ml})$. During the follow-up period, six patients (7\%) with osteoprotegerin levels below the median and 14 patients $(16 \%)$ with osteoprotegerin levels above the median died. The log rank test demonstrated a significant difference between the groups $(p=0.006)$. Furthermore, when applying Cox regression analysis high levels of osteoprotegerin revealed a fourfold increased risk for all-cause mortality 
Table 1 Baseline clinical and laboratory characteristics of 573 type 1 diabetic patients followed for $11.3(0.0-12.9)$ years according to nephropathy status

Data are $n$, mean \pm SD or median (range)

${ }^{a}$ Some patients with previously persistent macroalbuminuria receiving antihypertensive treatment had values $<300 \mathrm{mg} / 24 \mathrm{~h}$ at the time of investigation

SR, simplex retinopathy; PR, proliferative retinopathy

\begin{tabular}{llll}
\hline Characteristic & Nephropathy $(n=397)$ & Normoalbuminuria $(n=176)$ & $p$ value \\
\hline Sex (men/women) & $243 / 154$ & $105 / 71$ & 0.78 \\
Age (years) & $42.1 \pm 10.6$ & $42.6 \pm 9.7$ & 0.61 \\
Duration of diabetes (years) & $28.3 \pm 8.9$ & $27.6 \pm 8.3$ & 0.37 \\
BMI $\left(\mathrm{kg} / \mathrm{m}^{2}\right)$ & $24.29 \pm 3.4$ & $23.7 \pm 2.5$ & 0.036 \\
$\mathrm{HbA}_{1 \mathrm{c}}(\%)$ & $9.4 \pm 1.5$ & $8.5 \pm 1.1$ & $<0.001$ \\
Antihypertensive treatment (\%) & 75.0 & 9.7 & $<0.001$ \\
Insulin dose (IU) & $44.1 \pm 14.5$ & $40.4 \pm 13.0$ & 0.005 \\
Systolic BP (mmHg) & $145 \pm 22$ & $132 \pm 18$ & $<0.001$ \\
Diastolic BP (mmHg) & $82 \pm 12$ & $76 \pm 10$ & $<0.001$ \\
UAER (mg/24 h) & $609(10-14,545)^{\mathrm{a}}$ & $8(1-30)$ & - \\
Serum creatinine (mmol/l) & $102(52-684)$ & $76(53-116)$ & $<0.001$ \\
GFR (ml min $\left.{ }^{-1} 1.73 \mathrm{~m}{ }^{-2}\right)$ & $67 \pm 28$ & - & - \\
Serum cholesterol (mmol/l) & $5.6 \pm 1.2$ & $4.8 \pm 1.0$ & $<0.001$ \\
Serum HDL-cholesterol (mmol/l) & $1.5 \pm 0.7$ & $1.6 \pm 0.5$ & 0.001 \\
Serum triacylglycerol (mmol/l) & $1.3(0.3-9.9)$ & $0.8(0.3-3.0)$ & $<0.001$ \\
Smoking (\%) & 46.7 & 42.6 & 0.41 \\
Retinopathy (0/SR/PR) & $6 / 129 / 262$ & $62 / 95 / 19$ & $<0.001$ \\
Myocardial infarction (\%) & 4.0 & 1.1 & 0.07 \\
Stroke (\%) & 6.5 & 0.6 & $<0.001$ \\
NT-proBNP (ng/l) & $89.1(5-35000)$ & $27.8(5-453)$ & $<0.001$ \\
C-reactive protein (mg/l) & $1.2(0.1-52)$ & $0.9(0.1-47)$ & 0.002 \\
Osteoprotegerin ( $\mu \mathrm{g} / \mathrm{ml})$ & $3.0(1.4-11.4)$ & $2.6(1.4-8.2)$ & $<0.001$ \\
& & & \\
\hline
\end{tabular}

in relation to low levels (hazard ratio [HR] 4.10, 95\% CI $1.37-12.28 ; p=0.012$ ). However, this relationship disappeared after adjusting for baseline variables. Because of the low number of events in the normoalbuminuric group, further analyses are restricted to patients with diabetic nephropathy.

The 397 patients with diabetic nephropathy were divided into quartiles according to osteoprotegerin levels $(\leq 2.40$, $2.41-2.97,2.98-3.83, \geq 3.84 \mu \mathrm{g} / \mathrm{ml})$. Ten patients with osteoprotegerin levels in the first quartile $(10 \%), 21$ patients in the second quartile $(21 \%), 35$ patients in the third quartile (35\%) and 60 patients in the fourth quartile $(61 \%)$ died during follow-up. Figure 1 shows the Kaplan-Meier

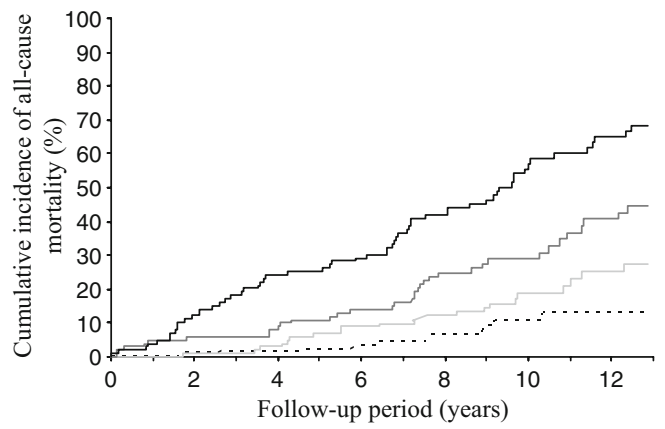

Fig. 1 Kaplan-Meier curves for all-cause mortality in 397 type 1 diabetic patients with diabetic nephropathy $(p<0.001)$. Dotted black line, first quartile of osteoprotegerin concentration; light grey line, second quartile; dark grey line, third quartile; solid black line, fourth quartile curve for overall mortality in patients with diabetic nephropathy $(p<0.001)$. An eightfold increased risk of all-cause mortality was found for high (fourth quartile) vs low (first quartile) levels of osteoprotegerin (HR 8.26 [4.23-16.15], $p<0.001)$. High osteoprotegerin remained an independent predictor of all-cause mortality in the Cox regression model (covariate-adjusted HR 3.00 [1.24-7.27], $p=0.015$ ) (Table 2). We then examined whether high levels of osteoprotegerin were associated with an increased risk of cardiovascular mortality. During follow-up, six patients with osteoprotegerin levels in the first quartile $(6 \%)$, eight patients in the second quartile $(8 \%), 28$ patients in the third quartile (28\%) and 39 patients in the fourth quartile (39\%) died of reasons related to cardiovascular disease. Figure 2 shows the Kaplan-Meier curve for cardiovascular mortality $(p<0.001)$. High levels of osteoprotegerin predicted an increased risk of cardiovascular mortality (HR 8.76 [3.7120.71], $p<0.001$; covariate-adjusted HR 4.88, [1.57-15.14], $p=0.006$ ) (Table 2). The same trend was seen when looking at non-fatal cardiovascular events $(n=84)$. High levels increased the risk of non-fatal cardiovascular events in a univariate proportional hazards regression analysis (HR 3.04 [1.45-6.35], $p=0.003$ ). However, this association disappeared when adjusting for confounding factors (covariateadjusted HR 1.21 [0.46-3.20], $p>0.05$ ).

Osteoprotegerin concentration was tested for a correlation with the well-known cardiovascular risk factors Creactive protein and NT-proBNP [27]. Osteoprotegerin concentration correlated with NT-proBNP $(r=0.56, p<0.001)$ 
Table 2 Hazard ratio of long-term cumulative mortality, cardiovascular mortality and ESRD after adjustment for confounding factors

\begin{tabular}{lcll}
\hline Parameter & Hazard ratio & $95 \%$ CI & $p$ value \\
\hline $\begin{array}{l}\text { All-cause mortality } \\
\text { Second vs first quartile }\end{array}$ & 1.83 & $0.79-4.22$ & 0.157 \\
$\quad$ Third vs first quartile & 2.15 & $0.93-4.98$ & 0.074 \\
$\quad \begin{array}{l}\text { Fourth vs first quartile } \\
\text { Cardiovascular mortality }\end{array}$ & 3.00 & $1.24-7.27$ & 0.015 \\
$\quad$ Second vs first quartile & 1.32 & $0.43-4.09$ & 0.631 \\
$\quad$ Third vs first quartile & 3.81 & $1.32-10.95$ & 0.013 \\
$\quad$ Fourth vs first quartile & 4.88 & $1.57-15.14$ & 0.006 \\
ESRD & & & \\
$\quad$ Second vs first quartile & 2.14 & $0.66-6.85$ & 0.202 \\
Third vs first quartile & 3.94 & $1.36-11.38$ & 0.011 \\
Fourth vs first quartile & 4.32 & $1.45-12.87$ & 0.009 \\
\hline
\end{tabular}

${ }^{\text {a }}$ Primary endpoints are adjusted for the following confounding factors: sex, age, smoking, $\mathrm{BP}, \mathrm{HbA}_{1 \mathrm{c}}$, GFR, serum cholesterol, UAER, antihypertensive treatment and a history of cardiovascular events at baseline

${ }^{\mathrm{b}}$ The secondary endpoint is adjusted for the following confounding factors: sex, age, systolic BP, $\mathrm{HbA}_{1 \mathrm{c}}$, serum cholesterol, GFR, UAER and antihypertensive treatment

but not with C-reactive protein $(r=0.02, p=0.73)$. The predictive value of osteoprotegerin remained unchanged if these markers were included in the analysis of cardiovascular mortality. The covariate-adjusted HR was 4.90 when comparing the fourth and first osteoprotegerin quartiles $(1.55-15.49, p=0.007)$. Similar trends were seen for other endpoints, but nevertheless the predictive value of osteoprotegerin was moderately reduced. In addition, evaluating osteoprotegerin as a continuous variable introduced only minor changes in the statistical output. Furthermore, when performing the same analyses for all patients $(n=573)$ adjusting for the presence or absence of nephropathy, similar results were seen.

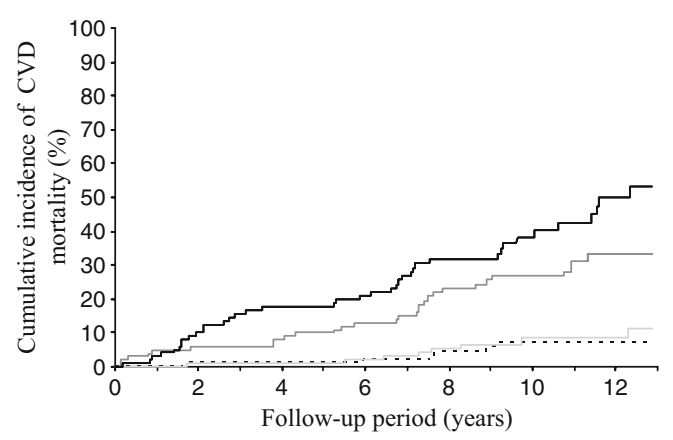

Fig. 2 Kaplan-Meier curves for fatal cardiovascular (CVD) events in 397 type 1 diabetic patients with diabetic nephropathy $(p<0.001)$. Dotted black line, first quartile of osteoprotegerin concentration; light grey line, second quartile; dark grey line, third quartile; solid black line, fourth quartile

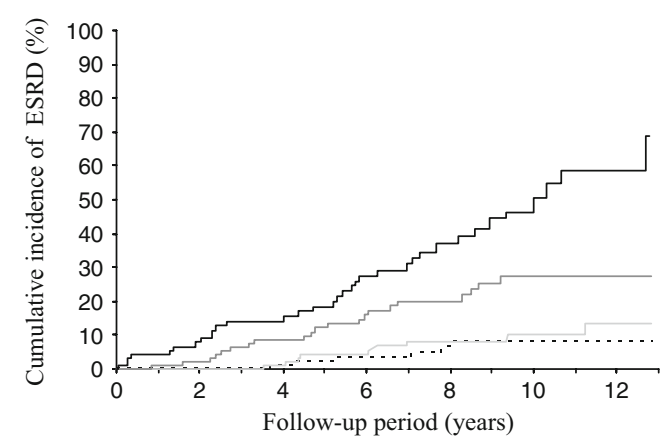

Fig. 3 Kaplan-Meier curves of ESRD in 397 type 1 diabetic patients with diabetic nephropathy $(p<0.001)$. Dotted black line, first quartile of osteoprotegerin concentration; light grey line, second quartile; dark grey line, third quartile; solid black line, fourth quartile

The overall prognostic value of osteoprotegerin for overall mortality was evaluated as the mean area under the receiver operating curve. For osteoprotegerin the AUC was $0.75(0.70-0.81)$. Furthermore, the AUCs for a risk score for the model containing other variables (sex, age, smoking, systolic $\mathrm{BP}, \mathrm{HbA}_{1 \mathrm{c}}$, serum cholesterol, GFR, UAER, antihypertensive treatment and history of cardiovascular events) with and without osteoprotegerin were $0.83(0.79-0.88)$ and $0.82(0.78-0.87)$, respectively.

Progression of nephropathy In the group of patients with diabetic nephropathy, progression to dialysis or transplantation was seen in six patients in the first quartile $(6 \%)$, nine patients in the second quartile (9\%), 21 patients in the third quartile $(21 \%)$ and 34 patients in the fourth quartile (35\%) (Fig. 3) $(p<0.001)$. Cox regression analysis revealed a ninefold increased risk of ESRD in patients with high osteoprotegerin levels in relation to low levels (HR 9.28 [3.89-22.14], $p<0.001)$. This relationship persisted after adjusting for confounding factors (covariate-adjusted HR 4.32 [1.45-12.87], $p=0.009$ ) (Table 2).

In addition, the rate of decline in kidney function was explored using annually measured GFR ( $n=386$ patients). The mean annual rate of decline in GFR differed significantly according to osteoprotegerin levels: first quartile 3.7 (4.2) $\mathrm{ml} \mathrm{min}^{-1} 1.73 \mathrm{~m}^{-2}$; second quartile 4.0 (3.1) $\mathrm{ml} \mathrm{min}^{-1}$ $1.73 \mathrm{~m}^{-2}$; third quartile $4.8(4.3) \mathrm{ml} \mathrm{min}^{-1} 1.73 \mathrm{~m}^{-2}$; fourth quartile 4.9 (4.4) $\mathrm{ml} \mathrm{min}^{-1} 1.73 \mathrm{~m}^{-2} ; p=0.015$. This effect persisted after correction for well-known progression promoters $(p=0.017)$.

\section{Discussion}

This prospective observational follow-up study revealed that plasma osteoprotegerin concentration is a novel independent risk marker for all-cause and cardiovascular mortality in type 
1 diabetic patients with overt nephropathy. Moreover, we found that the plasma osteoprotegerin concentration predicts deterioration of kidney function. These predictive capacities persisted after adjustment for conventional cardiovascular and renal risk factors, including GFR.

Osteoprotegerin in the arterial wall Osteoprotegerin is a $120 \mathrm{kDa}$ secretory glycoprotein belonging to the TNF receptor superfamily. It was first discovered in bone [1] but is also present in large amounts in the arterial wall [4]. Osteoprotegerin may be involved in the development of vascular calcifications, since immunohistochemical studies have shown co-localisation of osteoprotegerin and areas of calcification in media and atherosclerotic plaques [28]. Experimental studies have shown associations between vascular calcification in vitro and osteoprotegerin production [9]. In addition, osteoprotegerin has also been suggested to be a survival factor for endothelial cells [29]. Nevertheless, osteoprotegerin knockout mice develop vascular calcifications [30].

Osteoprotegerin and diabetes As previously shown, osteoprotegerin accumulates in aorta from patients with type 1 and type 2 diabetes [4]. This accumulation is seen in the tunica media from areas of the tissue with and without atherosclerotic plaques. The accumulation of osteoprotegerin throughout the deeper layers of the vessel wall may reflect generalised changes in the arterial system in diabetes, as part of diffuse arterial changes such as alterations in glycoproteins [31], collagens [32] and glycosaminoglycans [33]. In addition, osteoprotegerin may be related to the development of medial calcification, which is frequently present in patients with diabetes [34, 35]. Two recent experimental studies have confirmed the finding of increased expression of the gene encoding osteoprotegerin and production of the protein in arteries from animals with experimental diabetes $[10,11]$. At a first glance, it may seem contradictory that osteoprotegerin - a putative beneficial calcification inhibitor-accumulates in the arterial system in diabetes and that a high concentration of plasma osteoprotegerin is a cardiovascular risk marker. However, osteoprotegerin accumulation may be part of an osteogenic differentiation of vascular cells, as a compensatory response against calcification [36]. A series of other factors related to vascular calcification, such as vitamin D, BMP2, fetuin, matrix $\gamma$-carboxyglutamic acid (Gla) protein and osteopontin may also be relevant to this context. However, there is no information about these factors in relation to diabetic macrovascular disease. Alternatively, alterations in arterial osteoprotegerin amounts in diabetes may reflect direct effects of hormonal and inflammatory factors, since osteoprotegerin production can be influenced by both TNF- $\alpha$ and insulin in vascular smooth muscle cells in vitro [4].
A recent study has observed that osteoprotegerin is strongly upregulated at the RNA level in kidney samples from patients with diabetes [37]. This is interesting in relation to our observation that osteoprotegerin concentration predicts ESRD.

Plasma osteoprotegerin and cardiovascular risk Plasma osteoprotegerin concentration correlates to both diabetes and cardiovascular disease in cross-sectional studies. This was first shown by Browner et al. [12], who reported that although no associations were seen between the bone parameters and plasma osteoprotegerin levels, individuals with diabetes as well as persons with cardiovascular disease had increased values. Since then, these findings have been confirmed in both type $1[38,39]$ and type 2 diabetes [13, 40]. Moreover, we recently found that plasma osteoprotegerin is increased in subgroups of type 1 diabetic patients with nephropathy and in subgroups with signs of cardiovascular disease [18]. In line with the suggestion that osteoprotegerin is an inhibitor of vascular calcification, it is interesting that plasma osteoprotegerin is associated with arterial calcification in humans, as estimated by different modern imaging techniques [16, 41]. Only a few prospective studies on plasma osteoprotegerin as a putative predictor of cardiovascular events have been published. Anand et al. found in a study of 510 type 2 diabetic individuals that high levels of plasma osteoprotegerin predicted cardiovascular events over a relative short observation period of approximately 18 months [19]. Moreover, in post-kidney-transplant non-diabetic patients at high cardiovascular risk, serum osteoprotegerin was recently shown to predict long-term patient survival and cardiovascular mortality very well [20]. Our finding of a strong predictive effect of plasma osteoprotegerin in type 1 diabetic individuals with nephropathy supports the finding that the circulating osteoprotegerin level reflects arterial osteoprotegerin accumulation, as part of a generalised arteriopathy related to calcifications. Alternatively, the predictive value of osteoprotegerin may relate to a possible association to heart failure, which a recent study indicated [42]. Although data on heart failure are not available in the present study, our finding of an association between osteoprotegerin and NT-proBNP supports this notion.

Conclusion Our data show that plasma osteoprotegerin is a strong and independent predictor of all-cause and cardiovascular mortality as well as for deterioration of kidney function in patients with nephropathy. One explanation may be that osteoprotegerin accumulates in the vascular wall in diabetes as part of the macroangiopathy. High plasma levels may thus reflect the degree of macrovascular disease and consequently predict cardiovascular disease. Our present findings point to the clinical use of plasma osteoprotegerin for future risk stratification of these patients. 
Acknowledgements The EURAGEDIC study was supported by the European Commission (contract QLG2-CT-2001-01669), the NOVO Nordisk Research Foundation and the Danish Medical Research Council. We acknowledge the assistance of K. Hald, B. V. Hansen, A.-K. Holstein, B. R. Jensen, T. R. Juhl, K. Mathiassen, L. Pietraszek and U. M. Smidt. Roche Diagnostics, Germany provided the kits and measured NT-proBNP and C-reactive protein.

Duality of interest The authors declare that there is no duality of interest associated with this manuscript.

\section{References}

1. Simonet WS, Lacey DL, Dunstan CR et al (1997) Osteoprotegerin: a novel secreted protein involved in the regulation of bone density. Cell 89:309-319

2. Tsuda E, Goto M, Mochizuki S et al (1997) Isolation of a novel cytokine from human fibroblasts that specifically inhibits osteoclastogenesis. Biochem Biophys Res Commun 234:137-142

3. Tomoyasu A, Goto M, Fujise N et al (1998) Characterization of monomeric and homodimeric forms of osteoclastogenesis inhibitory factor. Biochem Biophys Res Commun 245:382-387

4. Olesen P, Ledet T, Rasmussen LM (2005) Arterial osteoprotegerin: increased amounts in diabetes and modifiable synthesis from vascular smooth muscle cells by insulin and TNF-alpha. Diabetologia 48:561-568

5. Schoppet M, Al Fakhri N, Franke FE et al (2004) Localization of osteoprotegerin, tumor necrosis factor-related apoptosis-inducing ligand, and receptor activator of nuclear factor-kappaB ligand in Monckeberg's sclerosis and atherosclerosis. J Clin Endocrinol Metab 89:4104-4112

6. Emery JG, McDonnell P, Burke MB et al (1998) Osteoprotegerin is a receptor for the cytotoxic ligand TRAIL. J Biol Chem 273:14363-14367

7. Malyankar UM, Scatena M, Suchland KL, Yun TJ, Clark EA, Giachelli CM (2000) Osteoprotegerin is an alpha vbeta 3-induced, NF-kappa B-dependent survival factor for endothelial cells. J Biol Chem 275:20959-20962

8. Schoppet M, Preissner KT, Hofbauer LC (2002) RANK ligand and osteoprotegerin: paracrine regulators of bone metabolism and vascular function. Arterioscler Thromb Vasc Biol 22:549-553

9. Olesen P, Nguyen K, Wogensen L, Ledet T, Rasmussen LM (2007) Calcification of human vascular smooth muscle cells: associations with osteoprotegerin expression and acceleration by high-dose insulin. Am J Physiol Heart Circ Physiol 292:H1058-H1064

10. Heinonen SE, Leppänen P, Kholová I et al (2007) Increased atherosclerotic lesion calcification in a novel mouse model combining insulin resistance, hyperglycemia and hypercholesterolemia. Circ Res 101:1058-1067

11. Vaccarezza M, Bortul R, Fadda R, Zweyer M (2007) Increased OPG expression and impaired OPG/TRAIL ratio in the aorta of diabetic rats. Med Chem 4:387-391

12. Browner WS, Lui LY, Cummings SR (2001) Associations of serum osteoprotegerin levels with diabetes, stroke, bone density, fractures, and mortality in elderly women. J Clin Endocrinol Metab 86:631-637

13. Knudsen ST, Foss CH, Poulsen PL, Andersen NH, Mogensen CE, Rasmussen LM (2003) Increased plasma concentrations of osteoprotegerin in type 2 diabetic patients with microvascular complications. Eur J Endocrinol 149:39-42

14. Schoppet M, Sattler AM, Schaefer JR, Herzum M, Maisch B, Hofbauer LC (2003) Increased osteoprotegerin serum levels in men with coronary artery disease. J Clin Endocrinol Metab 88:1024-1028
15. Jono S, Ikari Y, Shioi A et al (2002) Serum osteoprotegerin levels are associated with the presence and severity of coronary artery disease. Circulation 106:1192-1194

16. Clancy P, Oliver L, Jayalath R, Buttner P, Golledge J (2006) Assessment of a serum assay for quantification of abdominal aortic calcification. Arterioscler Thromb Vasc Biol 26:2574-2576

17. Dellegrottaglie S, Sanz J, Rajagopalan S (2006) Molecular determinants of vascular calcification: a bench to bedside view. Curr Mol Med 6:515-524

18. Rasmussen LM, Tarnow L, Hansen TK, Parving HH, Flyvbjerg A (2006) Plasma osteoprotegerin levels are associated with glycaemic status, systolic blood pressure, kidney function and cardiovascular morbidity in type 1 diabetic patients. Eur $\mathrm{J}$ Endocrinol 154:75-81

19. Anand DV, Lahiri A, Lim E, Hopkins D, Corder R (2006) The relationship between plasma osteoprotegerin levels and coronary artery calcification in uncomplicated type 2 diabetic subjects. J Am Coll Cardiol 47:1850-1857

20. Hjelmesaeth J, Ueland T, Flyvbjerg A et al (2006) Early posttransplant serum osteoprotegerin levels predict long-term (8year) patient survival and cardiovascular death in renal transplant patients. J Am Soc Nephrol 17:1746-1754

21. Ueland T, Jemtland R, Godang K et al (2004) Prognostic value of osteoprotegerin in heart failure after acute myocardial infarction. J Am Coll Cardiol 44:1970-1976

22. Parving H-H, Mauer M, Ritz E (2004) Diabetic nephropathy. In: Brenner BM (ed) Brenner and Rector's the kidney, 7th edn. Saunders, Boston, pp 1777-1818

23. Tarnow L, Groop PH, Hadjadj S et al (2008) European rational approach for the genetics of diabetic complications EURAGEDIC: patient populations and strategy. Nephrol Dial Transplant 23:161-168

24. Feldt-Rasmussen B, Dinesen B, Deckert M (1985) Enzyme immunoassay: an improved determination of urinary albumin in diabetics with incipient nephropathy. Scand J Clin Lab Invest 45:539-544

25. Bröchner-Mortensen J, Rödbro P (1976) Selection of routine method for determination of glomerular filtration rate in adult patients. Scand J Clin Lab Invest 36:35-45

26. Pfeffer MA, Swedberg K, Granger CB et al (2003) Effects of candesartan on mortality and morbidity in patients with chronic heart failure: the CHARM-Overall programme. Lancet 362:759-766

27. Astrup A-S, Kim WY, Tarnow L et al (2008) Relation of left ventricular function, mass, and volume to NT-proBNP in type 1 diabetic patients. Diabetes Care 31:968-970

28. Tyson KL, Reynolds JL, McNair R, Zhang Q, Weissberg PL, Shanahan CM (2003) Osteo/chondrocytic transcription factors and their target genes exhibit distinct patterns of expression in human arterial calcification. Arterioscler Thromb Vasc Biol 23:489-494

29. Scatena M, Giachelli C (2002) The alpha(v)beta3 integrin, NFkappaB, osteoprotegerin endothelial cell survival pathway. Potential role in angiogenesis. Trends Cardiovasc Med 12:83-88

30. Bucay N, Sarosi I, Dunstan CR et al (1998) Osteoprotegerindeficient mice develop early onset osteoporosis and arterial calcification. Genes Dev 12:1260-1268

31. Takemoto M, Yokote K, Nishimura M et al (2000) Enhanced expression of osteopontin in human diabetic artery and analysis of its functional role in accelerated atherogenesis. Arterioscler Thromb Vasc Biol 20:624-628

32. Rasmussen LM, Ledet $T$ (1993) Aortic collagen alterations in human diabetes mellitus. Changes in basement membrane collagen content and in the susceptibility of total collagen to cyanogen bromide solubilisation. Diabetologia 36:445-453

33. Heickendorff L, Ledet T, Rasmussen LM (1994) Glycosaminoglycans in the human aorta in diabetes mellitus: a study of tunica media from areas with and without atherosclerotic plaque. Diabetologia 37:286-292 
34. Niskanen L, Siitonen O, Suhonen M, Uusitupa MI (1994) Medial artery calcification predicts cardiovascular mortality in patients with NIDDM. Diabetes Care 17:1252-1256

35. Lehto S, Niskanen L, Suhonen M, Ronnemaa T, Laakso M (1996) Medial artery calcification. A neglected harbinger of cardiovascular complications in non-insulin-dependent diabetes mellitus. Arterioscler Thromb Vasc Biol 16:978-983

36. Rasmussen LM, Ledet T (2005) Osteoprotegerin and diabetic macroangiopathy. Horm Metab Res 37(Suppl 1):90-94

37. Lorz C, Benito-Martin A, Boucherot A et al (2008) The death ligand TRAIL in diabetic nephropathy. J Am Soc Nephrol 19:904-914

38. Xiang GD, Sun HL, Zhao LS (2007) Changes of osteoprotegerin before and after insulin therapy in type 1 diabetic patients. Diabetes Res Clin Pract 76:199-206
39. Galluzzi F, Stagi S, Salti R et al (2005) Osteoprotegerin serum levels in children with type 1 diabetes: a potential modulating role in bone status. Eur J Endocrinol 153:879885

40. Xiang GD, Xu L, Zhao LS, Yue L, Hou J (2006) The relationship between plasma osteoprotegerin and endotheliumdependent arterial dilation in type 2 diabetes. Diabetes 55: 2126-2131

41. Abedin M, Omland T, Ueland $T$ et al (2007) Relation of osteoprotegerin to coronary calcium and aortic plaque (from the Dallas Heart Study). Am J Cardiol 99:513-518

42. Anand DV, Lim E, Darko D et al (2007) Determinants of progression of coronary artery calcification in type 2 diabetes role of glycemic control and inflammatory/vascular calcification markers. J Am Coll Cardiol 50:2218-2225 